\title{
Fundamental gap of molecular crystals via constrained density functional theory
}

\author{
Andrea Droghetti, ${ }^{1}$ Ivan Rungger, ${ }^{2}$ Chaitanya Das Pemmaraju, ${ }^{3}$ and Stefano Sanvito ${ }^{4}$ \\ ${ }^{1}$ Nano-Bio Spectroscopy Group and European Theoretical Spectroscopy Facility (ETSF), Universidad del Pais Vasco CFM \\ CSIC-UPV/EHU-MPC and DIPC, Av. Tolosa 72, E-20018 San Sebastian, Spain \\ ${ }^{2}$ Materials Division, National Physical Laboratory, Teddington, TW11 OLW, United Kingdom \\ ${ }^{3}$ Chemical Sciences Division and Materials Sciences Division, Lawrence Berkeley National Laboratory, Berkeley, California 94720, USA \\ ${ }^{4}$ School of Physics, AMBER and CRANN Institute, Trinity College, Dublin 2, Ireland
}

(Received 22 February 2016; published 16 May 2016)

\begin{abstract}
The energy gap of a molecular crystal is one of the most important properties since it determines the crystal charge transport when the material is utilized in electronic devices. This is, however, a quantity difficult to calculate and standard theoretical approaches based on density functional theory (DFT) have proven unable to provide accurate estimates. In fact, besides the well-known band-gap problem, DFT completely fails in capturing the fundamental gap reduction occurring when molecules are packed in a crystal structures. The failure has to be associated with the inability of describing the electronic polarization and the real space localization of the charged states. Here we describe a scheme based on constrained DFT, which can improve upon the shortcomings of standard DFT. The method is applied to the benzene crystal, where we show that accurate results can be achieved for both the band gap and also the energy level alignment.
\end{abstract}

DOI: 10.1103/PhysRevB.93.195208

\section{INTRODUCTION}

Molecular crystals have attracted a large amount of interest in both basic and applied science [1]. Among their most important properties is the fundamental gap $E_{\text {gap }}$, defined as the energy difference between the ionization potential $(I)$ and the electron affinity $(A)$, i.e., $E_{\text {gap }}=I-A$. Although for molecules in the gas phase $E_{\text {gap }}$ is typically several $\mathrm{eV}$, this is drastically reduced in solids, because of the electrostatic polarization of the crystal [2-5]. According to the classical picture, an added charge localized on a molecule causes all surrounding molecules in the crystal to re-arrange their charge densities. Such rearrangement generates net dipole moments that orient so to close the electric field lines of that charge. As the result of this, $I$ decreases, while $A$ increases. An analogous effect has been also demonstrated experimentally for molecules adsorbed on polarizable substrates [6-9]. Here, an image charge, opposite to that of the molecule, is formed on the surface, and the attractive Coulomb interaction between the added hole/electron and the image charge results in the overall reduction of the fundamental gap. Notably, it is this reduced fundamental gap, which is relevant for applications involving charge transport, while the gas-phase fundamental gap is too large for any practical use.

From the theoretical perspective, the $G W$ approximation of many-body perturbation theory [10] has proven quite accurate in computing $E_{\text {gap }}$ and in accounting for strong polarization effects both at surfaces $[11,12]$ and in bulk crystals $[13,14]$. Unfortunately, $G W$ is still a computationally very expensive method, which cannot be routinely applied to crystals with unit cells typically containing several hundreds atoms. In contrast, density functional theory (DFT) may be seen as a much less demanding alternative, with the occupied Kohn-Sham (KS) eigenstates representing a good zeroth-order approximation to the real occupied quasiparticle states (see Ref. [15] for a detailed discussion about this issue). However, one has to face several problems when calculating $E_{\text {gap }}$ as the difference between the KS lowest unoccupied and highest occupied molecular orbital (LUMO and HOMO, respectively) energies, $E_{\mathrm{gap}}^{\mathrm{KS}}=e_{\mathrm{LUMO}}^{\mathrm{KS}}-e_{\mathrm{HOMO}}^{\mathrm{KS}}$. First, this procedure results in a considerable underestimation of $E_{\text {gap }}$ already for molecules in the gas phase. This is the infamous "band-gap problem" of DFT that affects the computation of the fundamental gap of all materials, organic as well as inorganic. Second, for the specific case of molecular crystals and for calculations performed with the available approximated exchange-correlation density functionals, $E_{\mathrm{gap}}^{\mathrm{KS}}$ does not capture any effect induced by the polarization of the surrounding environment $[11,12,14]$. This means that $E_{\text {gap }}^{\mathrm{KS}}$ is returned the same for the gas phase molecule and its crystal. A fortuitous error cancellation between the gap underestimation and the neglect of the environment polarization may sometimes happen in practice, but this is not generally the case, which makes quantitative predictions for new materials impossible. Corrective approaches are therefore required, based on a detailed understanding of the origin of these shortcomings of DFT.

The band-gap problem is related to the physical interpretation of the KS eigenenergies as excitation energies. In exact DFT, the "ionization potential theorem" [16-19] establishes the mathematical equality between the KS HOMO energy and the negative of the ionization potential, which means that $e_{\mathrm{HOMO}}^{\mathrm{KS}}=-I$. In contrast, no analogous theorem exists for the KS LUMO energy. This differs from the opposite of the electron affinity by a number equal to the derivative discontinuity $\Delta_{\mathrm{xc}}$ of the exchange-correlation functional at integer electron occupation [20-28], a quantity which is not known. Therefore, the fundamental gap and the KS gap do not coincide and $E_{\text {gap }}=E_{\text {gap }}^{\mathrm{KS}}+\Delta_{\mathrm{xc}}$. The difference becomes even larger when approximate functionals are used in DFT. In fact one has $E_{\text {gap }}=E_{\mathrm{gap}}^{\mathrm{KS}}+\Delta_{\mathrm{xc}}^{f}+\Delta_{s}^{f}$ [23], with $\Delta_{\mathrm{xc}}^{f}$ the derivative discontinuity of the approximate functional and $\Delta_{S}^{f}$ determined by the deviation of the approximate DFT energy from the exact linear dependence on the electron number between two consecutive integer occupations [18,24]. For the local density approximation (LDA) and the generalized 
gradient approximation (GGA) of the exchange-correlation functional [25], $\Delta_{\mathrm{xc}}^{f=\mathrm{LDA} / \mathrm{GGA}}$ spuriously vanishes, but one still has that $\Delta_{s}^{f=\mathrm{LDA} / \mathrm{GGA}}$ is not zero.

Nevertheless, for finite-size systems and, in particular, for small molecules in the gas phase, the fundamental gap can be accurately computed with the $\triangle \mathrm{SCF}$ method by finite-energy differences [26]. This, instead, is not true for extended systems, such as molecular crystals. In fact, because of the inherent LDA/GGA self-interaction error [27] leading to an excessive charge delocalization, a charge added to a system is incorrectly spread over the entire system in order to lower its total energy. As a result, the $\triangle \mathrm{SCF}$ gap is determined by the fractional charge error of the approximate functional [28] $\left(\Delta_{S}^{f}\right)$ and becomes equal to the KS gap [21,29] so that the two share the same error. This is the essence of the band-gap problem for extended systems in DFT with LDA/GGA [28,30,31].

While the derivative discontinuity and the band-gap problem are intrinsic to DFT even in its exact formulation (where the exact energy density functional is used) the deficiency in predicting the polarization-induced gap reduction is due to the utilization of functionals, where the correlation energy depends only on the local density and its gradient. In such functionals the exchange-correlation potential entering into the effective single-particle KS equations does not mimic any effect ascribable to nonlocal correlations, such as environmental screening. If the exact correlation functional was known, the calculated KS HOMO eigenenergy for the crystal would show the correct shift with respect to the gas phase value, in agreement with the ionization potential theorem, since exact DFT will return the exact $I$ for both systems. In contrast, the KS LUMO eigenenergy is not expected to be equal to the real excitation energy in any case, because of the derivative discontinuity.

A common approach to go around these problems of standard DFT consists in using hybrid functionals within the generalized-KS scheme (gKS) introduced by Seidl et al. [32]. In fact, the derivative discontinuity of the exact functional is expected to be small [32] and the delocalization error is mitigated [33]. Furthermore, an effective way to describe the fundamental gap reduction has been recently proposed by Rafaely-Abramson et al. [14]. These authors introduced a novel range-separated hybrid functional, called screened $\mathrm{RSH}$, where the short-range part of the exchange functional is treated in a semilocal approximation, while the long-range part is treated in a screened Fock-like manner, appropriate for incorporating the dielectric response of the crystal.

Here we propose an alternative and complementary approach, which is an extension of the $\triangle \mathrm{SCF}$ method and prevents the added charge from delocalizing over the entire system. As a consequence the computed fundamental gaps do not revert to the KS gaps. The critical observation is that in molecular crystals the hybridization between the various molecular units is rather weak, and each of these units is charge neutral, presenting an electron count identical to that of their gas phase. A molecular crystal thus appears naturally quite similar to a chain of distant atoms [29], and the fundamental gap can be described with finite total energy difference calculations by enforcing the localization of the added charge on a single molecule. In this way, one bypasses the $\mathrm{KS}$ band-gap problem and, at the same time, captures the response of the electron density of the surrounding molecules to the added charge. The calculations can be efficiently implemented by using the constrained DFT (CDFT). We note that, in contrast to the case of organic crystals, in inorganic semiconductors electrons are delocalized, and the considerations above do not hold [29]. An extension of the $\triangle \mathrm{SCF}$ method to treat inorganic semiconductors has been proposed by Chan and Ceder [34]. In their work they suggested adding a charge to a volume commensurate with the range of the dielectric screening effects determined from the extent of the LDA exchange-correlation hole. By studying approximately 100 different compounds, they have shown that the accuracy of the results rivals other state-of-art approaches, for instance, hybrid functionals and $G W$, while requiring much less computational resources.

In CDFT an appropriate external potential is introduced in the Kohn-Sham equations in order to implement the desired constraint on the charge density [35]. Importantly, the CDFT approach is fully variational, so that the energy minimum of the constrained functional represents the ground state of the system under that particular constraint [36-38]. CDFT has been used successfully for problems concerning molecular systems, for instance, for describing long-range charge-transfer excitations between molecules [36,39,40]. Moreover, in some recent works [41-43], it has been employed for determining the energy level alignment at hybrid organicinorganic interfaces. The results have been promising, showing that the method can capture image charge and polarization effects.

The goal of the present work consists in providing a welldefined procedure for the calculation of electron affinities, ionization potentials, and fundamental energy gaps of molecular crystals by means of CDFT. This is done by considering the prototypical example of the benzene crystal. The paper is organized as follows: We first describe the computational approach and the methods used in the calculations. Then, we present their application and the results. Finally we conclude mentioning some possible directions for further studies and for additional methodological improvements.

\section{COMPUTATIONAL METHODS}

Calculations have been performed by using a development version of the DFT package SIESTA [44], which includes an implementation of the CDFT method [42]. Norm-conserving Troullier-Martin pseudopotentials have been employed together with a basis set of double-zeta plus polarization quality. In order to compare our results to those obtained by Rafaely-Abramson et al. [14] with $G W$ and the screened-RSH functional, we have used the same unit cell considered in that work. The lattice vectors have been fixed to the experimental values, while the atomic coordinates inside the cells have been optimized with the Perdew-Zunger (PZ) parametrization of the LDA [45].

The benzene crystal has an orthorhombic unit cell containing four molecules and with lattice parameters $a=7.44 \AA$, $b=9.55 \AA$, and $c=6.92 \AA$ (see Fig. 1). All computed energy differences are converged up to $5 \mathrm{meV}$ with respect to the number of $\mathbf{k}$ points. The CDFT calculations have also been performed with PZ-LDA. In our implementation, the 


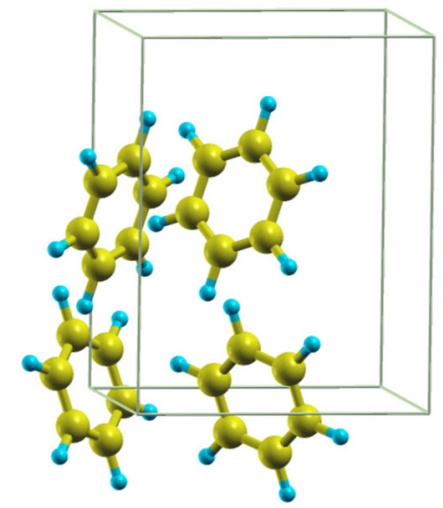

FIG. 1. The orthorhombic unit cell of the benzene crystal containing four molecules. Color code as follows: $\mathrm{C}$ atoms, yellow; $\mathrm{H}$ atoms, cyan.

constraint is applied by requiring that the total charge projected onto a given set of basis orbitals is equal to a specified value. We utilize the Löwdin projection scheme [46], which has been shown to give more accurate results than other considered schemes $[36,42]$.

In the $\triangle$ SCF method, the HOMO and LUMO energies of an isolated molecule are computed through total-energy differences,

$$
\begin{aligned}
& e_{\mathrm{HOMO}}=-I=E(N)-E(N-1), \\
& e_{\mathrm{LUMO}}=-A=E(N+1)-E(N),
\end{aligned}
$$

where $E(N)$ is the energy of the neutral molecule and $E$ $(N-1)$ and $E(N+1)$ are the energies of the corresponding cation and anion, respectively. Analogously, we calculate the HOMO and LUMO energies of molecular crystals by CDFT as

$$
\begin{aligned}
& e_{\mathrm{HOMO}}=E(N)-E_{\mathrm{CDFT}}(N-1), \\
& e_{\mathrm{LUMO}}=E_{\mathrm{CDFT}}(N+1)-E(N),
\end{aligned}
$$

where $E(N)$ is the total energy of the neutral cell and $E_{\mathrm{CDFT}}(N-1)$ and $E_{\mathrm{CDFT}}(N+1)$ are the total energies of the same cell with an added hole or electron, respectively. $E(N-1), E(N+1), E_{\mathrm{CDFT}}(N-1)$, and $E_{\mathrm{CDFT}}(N+1)$ are obtained via spin-polarized calculations.

In the computation of both the HOMO and the LUMO energies by CDFT, the additional charge is usually constrained to a single molecule. We note that for energies above the vacuum potential additional delocalized unbound states appear, usually termed as vacuum states [47]. If the molecular LUMO states have negative electron affinity, then the vacuum states will be lower in energy. Inclusion of such delocalized vacuum states within localized orbital basis sets calculations can be achieved by adding additional basis orbitals in the vacuum region [48]. However, vacuum states are neglected in the present study, which only concerns with molecular states.

The use of Eqs. (3) and (4) implies that one has to perform a calculation for a charged molecule in a periodic cell. However, the electrostatic energy of a periodically repeated system diverges (the total energy is not bound from below). In order to avoid this problem, it is customary to introduce a uniform charged jellium, which exactly neutralizes the net charge in the cell. As first noted by Leslie and Gillan [49], the energy of the new system then presents an additional contribution, which is equal to the electrostatic energy of a periodically repeated system containing a point charge $q$ immersed in the background jellium. Such additional contribution is just the Madelung energy, which writes

$$
E_{\mathrm{M}}=-\frac{q^{2} v_{\mathrm{M}}}{2 \epsilon}
$$

where $v_{\mathrm{M}}$ is the Madelung potential and $\epsilon$ is the macroscopic dielectric screening constant of the material (note that we use atomic units here). Since the Madelung energy represents a spurious addition to the system energy introduced by the computational methodology and due to the use of periodic boundary conditions, it must be removed from the calculated DFT total energy. Once this is done, the correct expressions for the HOMO and LUMO energies, Eqs. (3) and (4), read

$$
\begin{aligned}
e_{\mathrm{HOMO}}^{\mathrm{c}} & =E(N)-\left[E_{\mathrm{CDFT}}(N-1)-\left.E_{\mathrm{M}}\right|_{q=-1}\right], \\
e_{\mathrm{LUMO}}^{\mathrm{c}} & =\left[E_{\mathrm{CDFT}}(N+1)-\left.E_{\mathrm{M}}\right|_{q=1}\right]-E(N) .
\end{aligned}
$$

These equations show that the LUMO energy increases after the application of the Madelung correction, while, conversely, the HOMO energy decreases. This means that the corrected fundamental gap is larger than the uncorrected one.

For a cubic cell of lateral dimension $L$, the Madelung potential is available from literature $[49,50]$ and it is equal to $v_{\mathrm{M}}=\alpha / L$ with $\alpha=2.8373$. However, for the orthorhombic benzene unit cell, the Madelung potential $v_{\mathrm{M}}$ must be explicitly computed by using the Ewald summation [51,52],

$$
v_{\mathrm{M}}=\sum_{\mathbf{R}_{i}(i \neq 0)} \frac{\operatorname{erfc}\left(\eta\left|\mathbf{R}_{i}\right|\right)}{\left|\mathbf{R}_{i}\right|}+\frac{4 \pi}{\Omega} \sum_{\mathbf{G}_{i}(i \neq 0)} \frac{e^{-\frac{\left|\mathbf{G}_{i}\right|^{2}}{4 \eta^{2}}}}{\left|\mathbf{G}_{i}\right|^{2}}-\frac{2 \eta}{\sqrt{\pi}}-\frac{\pi}{\Omega \eta^{2}},
$$

where the first and second term are sums over all vectors with cell index $i \neq 0$ of the direct $\left(\left\{\mathbf{R}_{i}\right\}\right)$ and reciprocal $\left(\left\{\mathbf{G}_{i}\right\}\right)$ lattice. The rate of convergence of both sums is governed by the parameter $\eta$, which needs to be chosen so that they can be truncated without introducing any sizable error. This is done by imposing an error tolerance and a cutoff for the direct space sum and then by evaluating $\eta$ and the reciprocal space cutoff accordingly.

Once $v_{\mathrm{M}}$ has been obtained, the Madelung correction in Eq. (5) and, consequently, the corrected HOMO and LUMO energies can be calculated if the dielectric constant $\epsilon$ is known. The value of $\epsilon$ can be obtained from experimental data if available, or computed with the random phase approximation [53]. However, we here calculate $\epsilon$ by applying a finite size scaling approach, similar to that originally considered by Hine et $a$ l. in the study of defect formation energies in oxide insulators [54]. First, we compute the HOMO and LUMO energies, $e_{\mathrm{HOMO}}(\Omega)$ and $e_{\mathrm{LUMO}}(\Omega)$, for many supercells of different volume $\Omega$ by using Eqs. (3) and (4), which are now rewritten as

$$
\begin{aligned}
& e_{\mathrm{HOMO}}(\Omega)=E(\Omega, N)-E_{\mathrm{CDFT}}(\Omega, N-1), \\
& e_{\mathrm{LUMO}}(\Omega)=E_{\mathrm{CDFT}}(\Omega, N+1)-E(\Omega, N),
\end{aligned}
$$


and thus depend explicitly on $\Omega$. Then, for each supercell we plot $e_{\mathrm{HOMO}(\mathrm{LUMO})}(\Omega)$ as a function of Madelung potential $v_{\mathrm{M}}$ calculated by the Ewald summation. Finally we fit the corrected value of the HOMO (LUMO) energy $e_{\mathrm{HOMO}(\mathrm{LUMO})}^{\mathrm{c}}$ and the dielectric constant $\epsilon$ by using the linear equation,

$$
e_{\mathrm{HOMO}(\mathrm{LUMO})}(\Omega)=e_{\mathrm{HOMO}(\mathrm{LUMO})}^{\mathrm{c}}+(-) v_{\mathrm{M}} / 2 \epsilon,
$$

which is obtained from Eqs. (6)-(8) with $q^{2}=1$.

The procedure introduced here has several strengths. First, the dielectric constant is obtained without relying on any input from experiments or any theoretical approach other than CDFT. Second, we can inspect whether our results for $e_{\mathrm{HOMO}(\mathrm{LUMO})}(\Omega)$ versus $v_{\mathrm{M}}$ deviate from the linear behavior predicted by Eqs. (6) and (7), thus checking the reliability of the Madelung correction for the present problem. In fact, we note that the Madelung correction is expected to be adequate only for systems where the charge distribution can be approximated as pointlike. In contrast, for systems where this is not true (for instance, for charged defects in semiconductors [29]), different extensions of the Madelung correction have been discussed by Makov and Payne [55], as well as other authors [50,56,57].

\section{RESULTS}

Before investigating the molecular crystal, we evaluate the fundamental gap of the benzene molecule in the gas phase by using the $\triangle \mathrm{SCF}$ method. In order to explain how the DFT energies calculated by imposing periodic boundary conditions must be corrected for the Madelung contribution, we employ a supercell approach, where a single molecule is put inside a finite-sized box. In particular, here we consider several symmetric orthorhombic boxes of volume $\Omega_{n}=n^{3}(a b c)$ with $n=2, \ldots, 10$ and $a, b$, and $c$ being the lattice parameters of the benzene crystal unit cell. Then, by following the procedure outlined above, we compute the HOMO and LUMO energies according to Eqs. (1) and (2) for each system and plot the obtained values as a function of the corresponding Madelung potential $v_{\mathrm{M}}\left(\Omega_{n}\right)$. The results are presented in Fig. 2 and display an almost perfect linear trend for the HOMO (LUMO) energy, which decreases (increases) inversely to $v_{\mathrm{M}}\left(\Omega_{n}\right)$, or, alternatively, as a direct function of the supercell volume. We note that $v_{\mathrm{M}}\left(\Omega_{n}\right)=v_{\mathrm{M}}($ unit cell $) / n$, so that larger supercells correspond to smaller Madelung potentials. The fit of the data then provides $e_{\mathrm{HOMO}}^{\mathrm{c}}=-9.49 \mathrm{eV}$ and $e_{\mathrm{LUMO}}^{\mathrm{c}}=$ $1.83 \mathrm{eV}$, so that the gas phase benzene fundamental gap is $11.32 \mathrm{eV}$. These results for $e_{\mathrm{HOMO}}^{\mathrm{c}}$ and $e_{\mathrm{LUMO}}^{\mathrm{c}}$ are just slightly larger in magnitude than the experimental estimates, $e_{\mathrm{HOMO}}^{\exp }=-9.24 \mathrm{eV}$ and $e_{\mathrm{LUMO}}^{\exp }=1.14 \mathrm{eV}$, found, respectively, in Refs. [58,59]. Finally, we note that the computed dielectric constant (expressed in the system of atomic units) is $\epsilon=0.98$, which is very close to the expected vacuum dielectric constant $\epsilon_{0}=1$.

Next, we move to study the benzene crystal. The HOMO and LUMO energies are computed for supercells of different volumes by CDFT according to Eqs. (9) and (10). We can visualize directly the CDFT charge density of the added electron/hole and the consequent rearrangement of the charge density of the surrounding environment (Fig. 3). These rearrangements of the charge density computed by CDFT

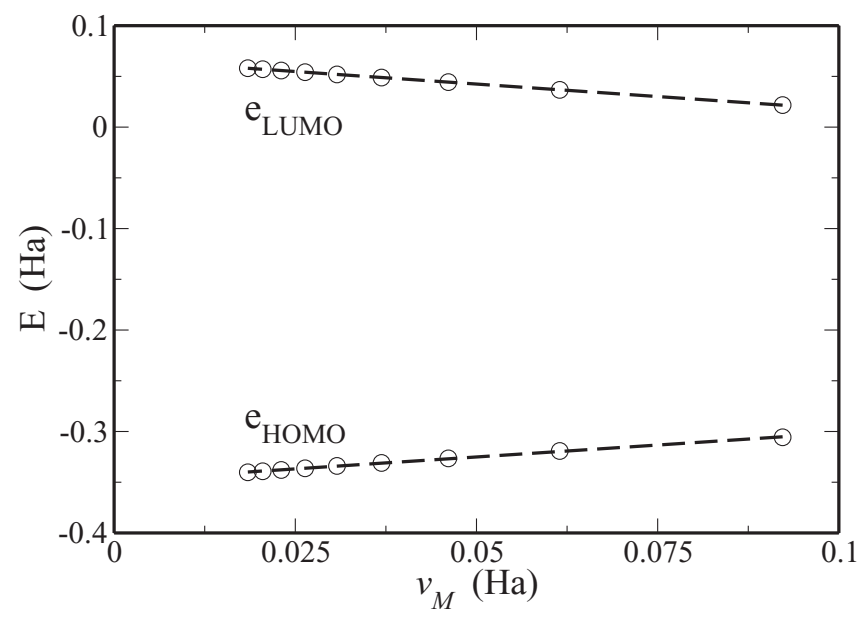

FIG. 2. HOMO and LUMO energies of gas phase benzene computed for several supercells with different Madelung potential $v_{\mathrm{M}}$. The energies are in Hartree for better display. Decreasing the Madelung potential is equivalent to increasing the supercell volume $\Omega_{n}$ as $v_{\mathrm{M}}\left(\Omega_{n}\right)=v_{\mathrm{M}}($ unit cell $) / n$. The data have been fitted through a straight line with Eq. (11).

represent the screening of the host crystal and therefore are of central importance for the subsequent evaluation of the fundamental gap. In Fig. 3 we clearly note that the presence of the electron/hole localized on one molecule induces the formation of a large dipole moment on the molecules of the first coordination shell. This effect, however, becomes already quite small for the second coordination shell and almost negligible for the third, showing that most of the screening happens in the first coordination shells.

Importantly, as pointed out by Hine et al. [54], the fitting procedure that we employ permits for the use of very asymmetric supercells constructed as an array of $l \times m \times n$ copies of the unit cell. This has the advantage that we can access a very large range of systems and, therefore, we can collect a large number of data to address the finite-size scaling problem. In contrast, if only symmetric $n \times n \times n$ supercells

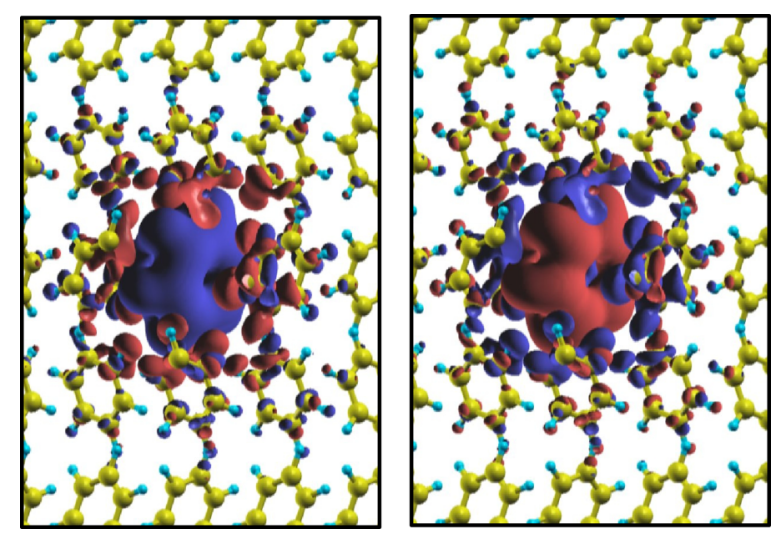

FIG. 3. Difference between the charge density of the neutral supercell and of the supercell with one extra hole (left panel) and on one extra electron (right panel) constrained on one molecule. Red (blue) isosurfaces represent positive (negative) values, i.e., charge accumulation (depletion). 


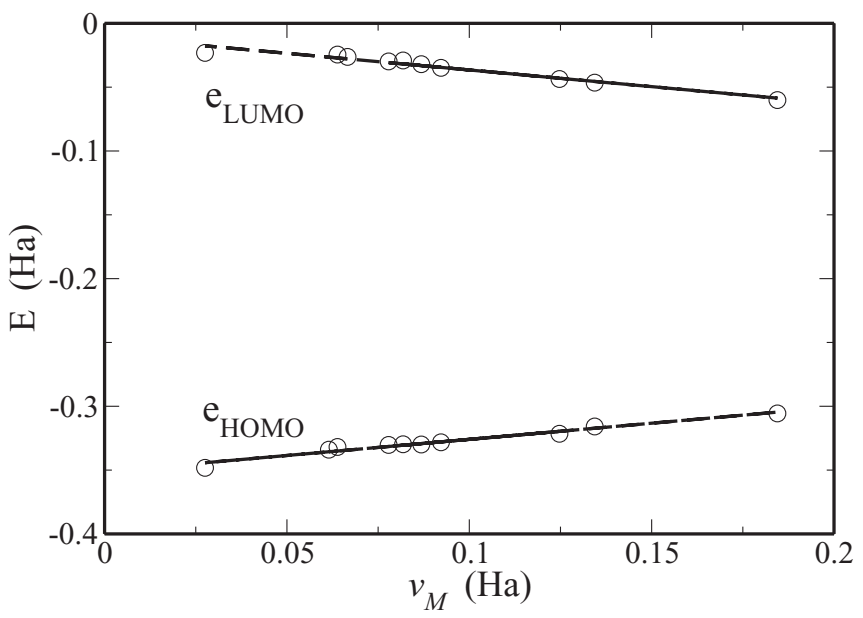

FIG. 4. HOMO and LUMO energies of the benzene crystal computed for several supercells with different Madelung potential $v_{\mathrm{M}}$. The energies are in Hartree for better display. The data have been been fitted through a straight line with Eq. (11).

were considered, as done in our calculations for the gas-phase molecule, just three data points for the HOMO and LUMO energies would be available, since the calculations become computationally unfeasible already for $n=4$.

The dependence of the HOMO and LUMO energies over the Madelung potential is presented in Fig. 4. As for the gas phase case, we find an almost perfect linear behavior of $e_{\mathrm{HOMO}}(\Omega)$ and $e_{\mathrm{LUMO}}(\Omega)$ against $v_{\mathrm{M}}$, with the absolute value of the correlation coefficient of the fit being equal to about 0.98 . This is very close to the maximal value of 1.0 for an exact linear dependence. Such excellent linear behavior also indicates that the dielectric properties of the benzene crystal are isotropic. If this was not the case, then the data obtained for supercells of different shapes would have shown a large spread, and only data for supercells constructed by repeating the same units along a specific crystallographic direction would have been on a straight line [60]. We remark here that our CDFT energies have an uncertainty of about $10-20 \mathrm{meV}$ with our calculation parameters.

After the linear extrapolation of the data of Fig. 4 we find a dielectric constant $\epsilon$ of 1.98 and 1.92 depending on whether the fit was performed for the HOMO or LUMO energy, respectively. Although these values somewhat underestimate the experimental one for (liquid) benzene [61,62], which is 2.28 , they are very close to the RPA value [14] of $\approx 2$. By extrapolating the data to an infinite-sized supercell we find $e_{\mathrm{HOMO}}^{\mathrm{c}}=-9.56 \mathrm{eV}$ and $e_{\mathrm{LUMO}}^{\mathrm{c}}=-0.28 \mathrm{eV}$ so that the fundamental gap decreases from that of the gas phase to $9.28 \mathrm{eV}$. This corresponds to a reduction of about $2 \mathrm{eV}$. Therefore, in the considered insulating molecular crystal, CDFT is demonstrated to capture nonlocal correlation effects due to the screening properties of the material. Analogous results have been found for molecules physisorbed on metallic surfaces [41,42], which shows that CDFT can correctly describe screening for both metallic and insulating materials.

Note that for all the methods used here (DFT KS eigenvalues, $\triangle \mathrm{SCF}$, and $\mathrm{CDFT}$ ) the effective vacuum energy for

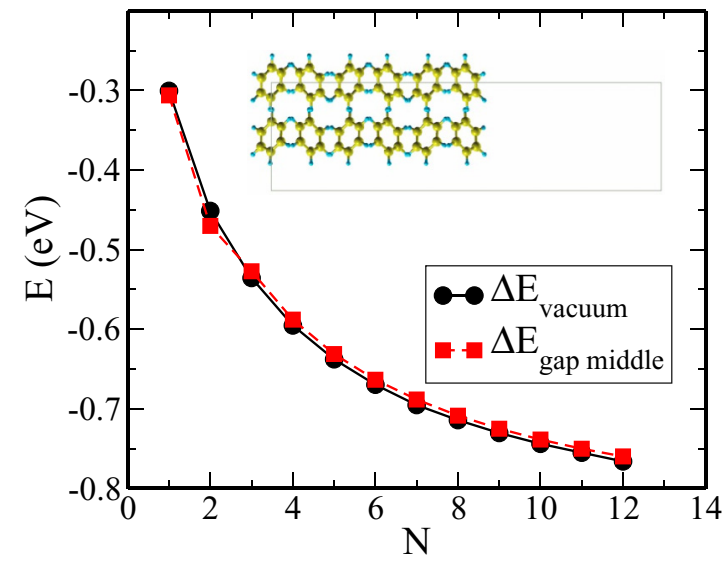

FIG. 5. Vacuum energy and middle of the KS gap for supercells, which contain slabs constructed by repeating $N$ benzene crystal unit cells along the nonperiodic direction (see inset, which displays one of such supercells for $N=3$ ). The vacuum energy is obtained from the Hartree potential in the middle of the vacuum region. The middle of the $\mathrm{KS}$ gap is calculated as $\left(e_{\mathrm{LUMO}}^{\mathrm{KS}}+e_{\mathrm{HOMO}}^{\mathrm{KS}}\right) / 2$. The lines interpolating the data are guides for eyes and not fitting curves.

the crystal needs to be removed from the calculated HOMO and LUMO energies in order to obtain the correct level alignment. This is necessary, since one needs to add to the DFT total energy of the charged system the energy from/to which the added/removed electron is transferred in a hypothetical experimental setup. Such energy is equal to the vacuum potential above the surface of the molecular crystal. We calculate such vacuum potential for the crystal by making a slab of increasing thickness, and evaluating the KS potential in the vacuum region. The results are displayed in Fig. 5, and show that for the considered system the vacuum potential decreases with increasing the thickness of the slab. Note that this shift in itself is arbitrary and depends on the used DFT code, since the potential of the entire cell is always defined only up to a constant. The only physically meaningful quantities are differences between eigenvalues or total energies with respect to this vacuum energy. In Fig. 5 we therefore also show the shift of the midpoint energy of the KS HOMO-LUMO gap with increasing number of layers, which for this system is essentially identical to the shift of the vacuum energy. This implies that for the crystal calculation the middle of the band gap for all methods needs to be at the same energy as for the gas phase, since its relative position with respect to the vacuum energy does not change.

The frontier energy levels for the benzene molecule in the gas phase and crystal form as obtained by directly looking at the DFT KS eigenvalues and by using $\triangle \mathrm{SCF}$ and CDFT, are summarized in Fig. 6. As outlined above, for this system the midpoint of the fundamental gap is at the same energy for both the isolated molecule and the crystal. As expected, we find that the KS gap drastically underestimates the fundamental gap for both the single molecule and the crystal. Even for the KS eigenvalues there is an apparent symmetric $0.3 \mathrm{eV}$ reduction of the KS HOMO and LUMO energies of the crystal compared to the KS HOMO and LUMO energies of the molecule. However, this is not due to polarization effects, but it results from the 


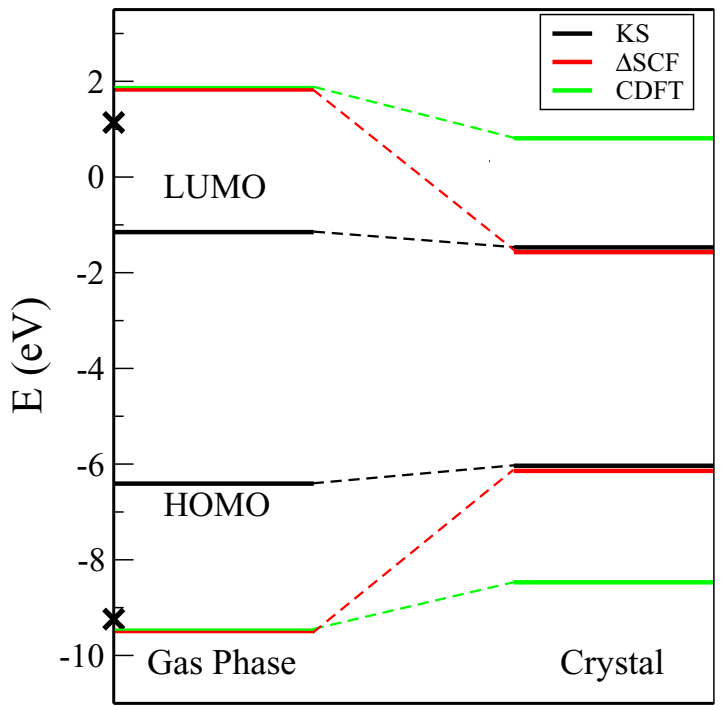

FIG. 6. HOMO and LUMO energies for gas phase and crystalline benzene. The results have been obtained by directly looking at the KS DFT eigenenergies and by using the $\triangle \mathrm{SCF}$ and the CDFT methods. In the case of $\triangle \mathrm{SCF}$ for the crystal, the displayed results have been computed for the largest supercell considered (i.e., $3 \times 3 \times 3$ ). The crosses are the experimental values for the gas phase molecule, while we have found no available data for the crystal.

energy dispersion of the KS band structure in the crystal. In fact, the KS HOMO and the LUMO energies of the benzene crystal are the top of the KS valence band and the bottom of the KS conduction band, respectively, both located at the $\Gamma$ point in the Brillouin zone (see Fig. 7). These bands have a finite width equal to about $0.6 \mathrm{eV}$, so that their top and bottom turn out to be displaced by about 0.3 around the band centers, which in turn are perfectly aligned with the isolated molecule KS HOMO and LUMO.

Furthermore, Fig. 6 shows that $\triangle \mathrm{SCF}$ performs well for the single molecule (note that for the gas phase $\triangle \mathrm{SCF}$ and CDFT are identical). However, when applied to the crystal,

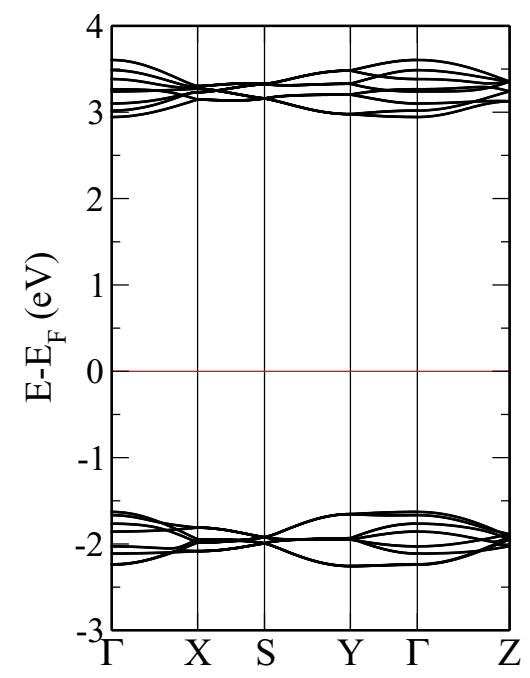

FIG. 7. KS valence and conduction band of the benzene crystal. the HOMO and LUMO energies turn out to be equal to the KS HOMO and LUMO energies (i.e., the top of the KS valence band and the bottom of the KS conduction band), and therefore $\triangle \mathrm{SCF}$ drastically underestimates the gap. In contrast, the CDFT HOMO and LUMO energies are symmetrically displaced, respectively, toward lower and higher energies when compared to the crystal KS values, so that the calculated CDFT gap becomes notably larger than the KS gap (Fig. 6). Most importantly, the CDFT crystal gap shows the expected reduction when compared to the gas phase one. Qualitatively correct results are therefore recovered only with CDFT. From a quantitative point of view, the assessment of the results is hindered by the lack of accurate experimental data for the crystal. We can however compare CDFT and $G W$ results (see Fig. 1 in Ref. [14]), and we observe that the CDFT gap is about $1 \mathrm{eV}$ smaller than the $G W$ one. This difference is already present for the gas phase results, while remarkably the size of gap reduction predicted by the two methods is in quite close quantitative agreement.

Despite the very satisfactory results obtained for the benzene crystal our method has a number of limitations, which are worth mentioning since one may encounter them when extending the procedure to other systems. The first limitation concerns the spatial distribution of the additional charge. In the case of the benzene crystal such charge is almost completely localized on a single molecular unit, but this may not always be the case for other materials. If the intermolecular hybridization is large, the constraint may not be well defined. As such enforcing localization on just one molecule will lead to a considerable systematic error. In these cases, one will need to develop a scheme for selecting the correct localization radius, which could follow from considerations about the screening length as proposed by Chan and Ceder in their work on inorganic materials [34]. A second consequence of such partial delocalization is that the Madelung corrections might not be always adequate and more advanced corrective schemes might be required. For instance, if one considers long polyacene compounds, where the added charge is likely to spread over the length of the molecule, the basic assumption that the added charge can be approximated as a pointlike one will break down. Finally, at a practical level we note that the method as presented here relies on several calculations for supercells of different sizes. Even though the use of asymmetric supercells effectively helps to limit the maximum size needed, CDFT will become computationally quite demanding for systems where already the unit cell contains many atoms.

\section{CONCLUSIONS AND PERSPECTIVES}

We have presented a well-defined computational scheme based on CDFT, which allows for the calculation of the ionization potentials and electron affinities, and consequently of the fundamental gap, of molecular crystals. As demonstrated for the case of benzene this scheme can potentially yield very accurate results, and most importantly capture effects due to electronic polarization.

We have shown that CDFT is a valuable alternative to $G W$ or screened-RSH approaches, since it provides a different and complementary approach to the calculation of the screening properties of organic materials and of the fundamental gap. 
In particular we expect CDFT to provide additional insight in systems with highly anisotropic screening. Similarly to those approaches the computational cost is rather large, mainly due to the calculations of larger supercells required in order to carry out the linear scaling analysis for the electrostatic correction. The main fundamental strength of the method is that it relies on DFT total energies rather than on the evaluation of KS eigenvalues. As such one can use local and semilocal functionals, which typically provide an accurate evaluation of the total energy.

\section{ACKNOWLEDGMENTS}

The authors are grateful to S. Refaely-Abramson for useful discussions. A.D. and I.R. were sponsored by the European Union through Project No. 618082 ACMOL. A.D. received additional support from the "Ministerio de Economia y Competitividad" (Mineco) of Spain (Grant No. FPDI-2013-16641). S.S. acknowledges the Quest project funded by European Research Council. Computational resources were provided by the Trinity Centre for High Performance Computing (TCHPC) and the Irish Centre for High-End Computing (ICHEC).
[1] J. D. Wright, Molecular Crystals, 2nd ed. (Cambridge University Press, Cambridge, 1995).

[2] N. Sato, K. Seki, and H. Inokuchi, J. Chem. Soc., Faraday Trans. 77, 1621 (1981)

[3] I. G. Hill, A. Kahn, Z. G. Soos, and R. A. Pascal, Jr., Chem. Phys. Lett. 327, 181 (2000).

[4] I. G. Hill, A. J. Mäkinen, and Z. H. Kafafi, J. Appl. Phys. 88, 889 (2000).

[5] A. Droghetti, M. Cinchetti, and S. Sanvito, Phys. Rev. B 89, 245137 (2014).

[6] R. Hesper, L. H. Tjeng, and G. A. Sawatzky, Europhys. Lett. 40, 177 (1997).

[7] J. Repp, G. Meyer, S. M. Stojkovic, A. Gourdon, and C. Joachim, Phys. Rev. Lett. 94, 026803 (2005).

[8] X. Lu, M. Grobis, K. H. Khoo, S. G. Louie, and M. F. Crommie, Phys. Rev. B 70, 115418 (2004).

[9] M. T. Greiner, M. G. Helander, W.-M. Tang, Z.-B. Wang, J. Qiu, and Z.-H. Lu, Nat. Mater. 11, 76 (2011).

[10] G. Onida, L. Reining, and A. Rubio, Rev. Mod. Phys. 74, 601 (2002).

[11] J. B. Neaton, M. S. Hybertsen, and S. G. Louie, Phys. Rev. Lett. 97, 216405 (2006).

[12] J. M. Garcia-Lastra, C. Rostgaard, A. Rubio, and K. S. Thygesen, Phys. Rev. B 80, 245427 (2009).

[13] S. Sharifzadeh, A. Biller, L. Kronik, and J. B. Neaton, Phys. Rev. B 85, 125307 (2012).

[14] S. Refaely-Abramson, S. Sharifzadeh, M. Jain, R. Baer, J. B. Neaton, and L. Kronik, Phys. Rev. B 88, 081204(R) (2013).

[15] L. Kronik and S. Kümmel, Top. Curr. Chem. 347, 137 (2014).

[16] M. Levy, J. P. Perdew and V. Sahni, Phys. Rev. A 30, 2745 (1984).

[17] C. O. Almbladh and U. vonBarth, Phys. Rev. B 31, 3231 (1985).

[18] J. P. Perdew, R. G. Parr, M. Levy, and J. L. Balduz, Phys. Rev. Lett. 49, 1691 (1982).

[19] J. P. Perdew and M. Levy, Phys. Rev. B 56, 16021 (1997).

[20] J. P. Perdew and M. Levy, Phys. Rev. Lett. 51, 1884 (1983).

[21] L. J. Sham and M. Schlüter, Phys. Rev. Lett. 51, 1888 (1983).

[22] P. Mori-Sanchez and A. J. Cohen, Phys. Chem. Chem. Phys. 16, 14378 (2014).

[23] A. J. Cohen, P. Mori-Sanchez, and W. Yang, Phys. Rev. B 77, 115123 (2008).

[24] W. Yang, Y. Zhang, and P. W. Ayers, Phys. Rev. Lett. 84, 5172 (2000).

[25] J. P. Perdew and S. Kurth, in A Primer in Density Functional Theory, edited by C. Fiolhais, F. Noguiera, and M. A. L.
Marques, Lecture Notes in Physics Vol. 620 (Springer, Heidelberg, 2003).

[26] R. O. Jones and O. Gunnarsson, Rev. Mod. Phys. 61, 689 (1989).

[27] J. P. Perdew and A. Zunger, Phys. Rev. B 23, 5048 (1981).

[28] P. Mori-Sanchez, A. J. Cohen, and W. Yang, Phys. Rev. Lett. 100, 146401 (2008).

[29] S. Lany and A. Zunger, Phys. Rev. B 78, 235104 (2008).

[30] C. S. Wang and W. E. Pickett, Phys. Rev. Lett. 51, 597 (1983).

[31] M. van Schilfgaarde, T. Kotani, and S. Faleev, Phys. Rev. Lett. 96, 226402 (2006).

[32] A. Seidl, A. Görling, P. Vogl, J. A. Majewski, and M. Levy, Phys. Rev. B 53, 3764 (1996).

[33] P. Mori-Sanchez, A. Cohen, and W. Yang, J. Chem. Phys. 125, 201102 (2006).

[34] M. K. Y. Chan and G. Ceder, Phys. Rev. Lett. 105, 196403 (2010).

[35] P. H. Dederichs, S. Blugel, R. Zeller, and H. Akai, Phys. Rev. Lett. 53, 2512 (1984).

[36] B. Kaduk, T. Kowalczyk, and T. Van Voorhis, Chem. Rev. 112, 321 (2012).

[37] Q. Wu and T. Van Voorhis, Phys. Rev. A 72, 024502 (2005).

[38] Q. Wu and T. Van Voorhis, J. Phys. Chem. A 110, 9212 (2006).

[39] Q. Wu and T. Van Voorhis, J. Chem. Phys. 125, 164105 (2006).

[40] S. Yeganeh and T. Van Voorhis, J. Phys. Chem. C 114, 20756 (2010).

[41] J. D. Sau, J. B. Neaton, H. J. Choi, S. G. Louie, and M. L. Cohen, Phys. Rev. Lett. 101, 026804 (2008).

[42] A. M. Souza, I. Rungger, C. D. Pemmaraju, U. Schwingenschloegl, and S. Sanvito, Phys. Rev. B 88, 165112 (2013).

[43] A. M. Souza, I. Rungger, R. B. Pontes, A. R. Rocha, A. J. Roque da Silva, U. Schwingenschlöegl, and S. Sanvito, Nanoscale 6, 14495 (2014).

[44] M. Soler, E. Artacho, J. D. Gale, A. Garcia, J. Junquera, P. Ordejon, and D. Sanchez Portal, J. Phys. Condens. Matter 14, 2745 (2002).

[45] J. P. Perdew and A. Zunger, Phys. Rev. B 23, 5048 (1981).

[46] P.-O. Löwdin, J. Chem. Phys. 18, 365 (1950).

[47] F. Picaud, A. Smogunov, A. Dal Corso, and E. Tosatti, Phys. Rev. B 65, 245105 (2002).

[48] C. Toher, I. Rungger, and S. Sanvito, Phys. Rev. B 79, 205427 (2009).

[49] M. Leslie and M. J. Gillan, J. Phys. C 18, 973 (1985).

[50] I. Dabo, B. Kozinsky, N. E. Singh-Miller, and N. Marzari, Phys. Rev. B 77, 115139 (2008).

[51] P. P. Ewald, Ann. Phys. 369, 253 (1921). 
[52] R. M. Martin, Electronic Structure. Basic Theory and Practical Methods (Cambridge University Press, Cambridge, 2004).

[53] E. Engel and R. M. Dreizler, Density Functional Theory: An Advanced Course (Springer-Verlag, Berlin/Heidelberg, 2011).

[54] N. D. M. Hine, K. Frensch, W. M. C. Foulkes, and M. W. Finnis, Phys. Rev. B 79, 024112 (2009).

[55] G. Makov and M. C. Payne, Phys. Rev. B 51, 4014 (1995).

[56] C. Freysoldt, J. Neugebauer, and C. G. Van de Walle, Phys. Rev. Lett. 102, 016402 (2009).

[57] H.-P. Komsa, T. T. Rantala, and A. Pasquarello, Phys. Rev. B 86, 045112 (2012).
[58] L. A. Chewter, M. Sander, K. Muller-Dethlefs, and E. W. Schlag, J. Chem. Phys. 86, 4737 (1987).

[59] J. C. Rienstra-Kiracofe, G. S. Tschumper, H. F. Schaefer, S. Nandi, and G. B. Ellison, Chem. Rev. 102, 231 (2002).

[60] S. T. Murphy and N. D. M. Hine, Phys. Rev. B 87, 09411 (2013).

[61] F. R. Goss, J. Chem. Soc., 888 (1940).

[62] F. Kohler, E. Liebermann, R. Schano, H. Affsprung, J. Morrow, K. Sosnkowskakehiaian, and H. Kehiaian, J. Chem. Thermodyn. 7, 241 (1975). 